\title{
Automatic classification of gait patterns using a smart rollator and the BOSS model
}

\author{
Maribel Ojeda $^{1}$, Atia Cortés ${ }^{2}$, Javier Béjar ${ }^{2}$, Ulises Cortés ${ }^{2}$ \\ ${ }^{1}$ Tecnológico de Monterrey - Campus Puebla Puebla, México \\ ${ }^{2}$ Universitat Politècnica de Catalunya - BarcelonaTech Barcelona, Catalunya, Spain \\ a01097403@itesm.mx, \{acortes, bejar, ia\}@cs.upc.edu,
}

\begin{abstract}
Nowadays, the risk of falling in older adults is a major concern due to the severe consequences it brings to socio-economic and public health systems. Some pathologies cause mobility problems in the aged population, leading them to fall and, thus, reduce their autonomy. Other implications of ageing involve having different gait patterns and walking speed. In this paper, a non-invasive framework is proposed to study gait in elder people using data collected by a smart rollator, the $i$-Walker. The analysis presented in this article uses a feature extraction method and a spectral embedding to represent the information and Bayesian clustering for the knowledge discovery. The algorithm considers raw data from the $i$-Walker sensors along with the calculated walking speed of each individual, which has been already used in clinical studies to assess physical and cognitive status of older adults. The results obtained demonstrate that the proposed analysis has the potential to separate in clusters the people of the two groups of interest: young people and geriatric.
\end{abstract}

\section{Introduction}

Gait and balance disorders are common in older adults and are the most significant cause of falls in this population sector. According to the Center for Disease Control and Prevention [CDC, 2016], falls are the leading cause of mortality in this segment; they can also lead to injury, causing loss of independence and critical health problems. Today, many countries are facing a rapidly ageing population. Worldwide, the number of this group was estimated to grow to more than two billion by 2050 [Nations, 2017]. The increased number of persons over 65 years will potentially lead to expensive health-care costs at the same time that a higher Quality of Life (QoL) is a significant concern for people.

Gait analysis is used to assess and treat individuals with conditions affecting their ability to walk. While studying elders' walking performance, it is noticeable that, eventually, this starts to decline to result in an inherent risk of falling. To put-on sight walking deficiencies, clinicians have developed several walking and cognitive tests to detect problems in older adults with injuries and diseases.

Two of the better-known assessments are the Ten Meter Walking Test and the Six Minute Walking Test (10MWT and $6 \mathrm{mWT}$ respectively from now on). The former has been previously used to predict the risk of falling or other health morbidities [Montero-Odasso et al., 2005]. The traditional 10MWT is measured with a stopwatch to monitor the time it takes to the patient to perform the distance, although the former and later two meters are discarded since they are considered as the acceleration and deceleration phases. In this work, participants performed an adaptation of $6 \mathrm{mWT}$, but reducing the time to three minutes due to clinical recommendations. Nevertheless, with these methods it is quite difficult to segment the exact time of the activity and, moreover, offers only a single variable of general information, which is the walking speed.

In recent years, the Information and Communication Technologies (ICT) had presented an enormous potential to promote the development of a series of devices, sensors and techniques that promote better evaluation measures and, thus, contribute to provide a more reliable and complete information about a patient. It is expected that ICT will we able to provide new tools of early detection and prevention of diseases in the elder population. The assistive device presented here (see Section 2.1) has been designed with the aim of helping physicians detecting certain kind of physical and cognitive conditions of a person by combining its sensors data along with the clinical information of an individual. In this respect, something as simple as identifying walking pattern could be used as an indicator of health.

In this work, we present an unsupervised approach to categorize elder individual's walking patterns into multiple groups among a population of volunteers with diverse ages based on their interaction with the $i$-Walker, which has been used as a measuring tool. The methodology developed is an efficient system for determining a person's walking-age.

The results of the presented work cluster the population into four categories in relation to their gait characteristics. The first two mainly separates the young people with higher and lower speed. The other two identify the geriatric gait and split the sample also considering the speed.

The paper is organized as follows: Section 2 introduces related work and the $i$-Walker; Section 3 presents the approach 


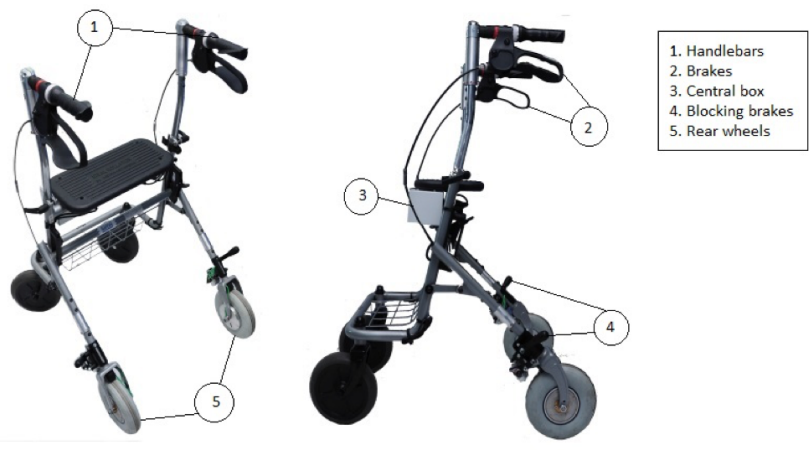

Figure 1: $i$-Walker components. The handlers contain the force sensors. The rear wheels have embed motors that work as actuators. The central box contains the computing power on- board. Blocking brakes provide a braking help when a downhill is detected.

used in this work, and Section 4 shows the experimental results. Lastly, Section 5 concludes this paper.

\section{Related Work}

Several ICT tools and machine learning procedures had been used for the study walking patterns, each of them with different advantages and disadvantages mainly related to the costs and the reliability of the results. This chapter present some of these proposals as well as the previous work made with the $i$-Walker.

\subsection{The i-Walker}

The $i$-Walker is an assistive device based on a standard 4wheeled rollator with embedded sensors and actuators. Fig 1 shows the $i$-Walker components, which have already been described in [Cortés et al., 2012].

The most innovative feature of the $i$-Walker is the design of the handlebars, which include embedded force sensors able to measure the longitudinal, lateral and vertical forces $(X, Y$ and $Z$ respectively) applied by the user when walking. The $i$-Walker has been previously integrated as part of post-stroke rehabilitation presented in [Giuliani et al., 2012], improving the outcomes obtained with traditional rehabilitation. In [Barban et al., 2017] participants of the EU funded project I-DONT-FALL [IDF, 2010] used the $i$-Walker as an assistive device that would help them reducing the number of falls as well as the risk and fear of falling after a three-months period of physical and/or cognitive training. In addition, authors in [Cortés et al., 2016] developed a prediction model able to detect people with major risk of falling after this training process.

In [Ballesteros et al., 2016], authors developed a system to assess the estimation error of the force sensors. The results showed an error lower than $10 \%$. This study also confirms the relationship between the force sensor and the support of the individual.

In [Moreno et al., 2015], authors proposed a methodology for detecting common walking patterns in the elderly according to their physical and cognitive conditions by using the Symbolic Aggregation Approximation (SAX) algorithm for time series representation. They used a sample of 200 older adults where some of them have had suffered one or more falls during the last year, while the others did not. The algorithm was able to separate between fallers and non fallers using hierarchical clustering.

\subsection{Related Work using other systems}

This section presents a description of previous research work in the field of gait analysis.

In 2014, [Jin et al., 2014] also used a 3-D accelerometer and a gyroscope to measure speed, acceleration, and tilting of the legs. They constructed a walking pattern database from 79 volunteers of ages ranging from 10 to 83 years old. Their proposed approach was to use intrinsic mode functions (IMF) as a representation method and K-means as the clustering technique. They were able to identify three groups, children of ages 10 and below, adults in 20-60s, and elders in 70s and 80 s.

In 2017, [Dolatabadi et al., 2017] used a system called GAITRite to gather the information from a sample of people of 88 adults, where 68 of them had suffered a stroke and 20 were healthy people. They used the allocated indexing membership (AIM) as a representation method and also the Bayesian Gaussian Mixture as the clustering technique. The results found three clusters: the first group was formed by the healthy people, group 2 had the worse condition and group 3 contained people with conditions but with improvements in their recovery.

\section{Approach}

This section introduces the data collection and methodology used to develop this work.

\subsection{Data collection}

Walking tests were conducted to record the data of the walking patterns of a group of volunteers. To perform the test, a total of 42 individuals whose ages range from 22 to 94 years were selected for this study. Each of them performed the $3 \mathrm{mWT}$ on a flat hallway moving back and forth. The data was collected by the $i$-Walker and sent to a computer using Matlab/Simulink. The variables are collected as time-series every $100 \mathrm{~ms}$. Several metrics were obtained, but the most important are: $(i)$ the vertical, longitudinal and transversal forces of both hands to measure the interaction between the participant and the device, (ii) the angular velocity to determine the end of a straight line, and (iii) the estimated pose in $X$ and $Y$.

The forces exerted on the handlebars were used as the input of the model on which the clustering performs the pattern recognition. In addition, the angular velocity and estimated pose were used to assess each individual's speed and to calculate the walked distance.

\subsection{Methodology}

The methodology comprises the following steps: representation and indexing, similarity measure, clustering, and evaluation.

The BOSS model is used for the representation and indexing. This method transforms the numeric time-series into a 


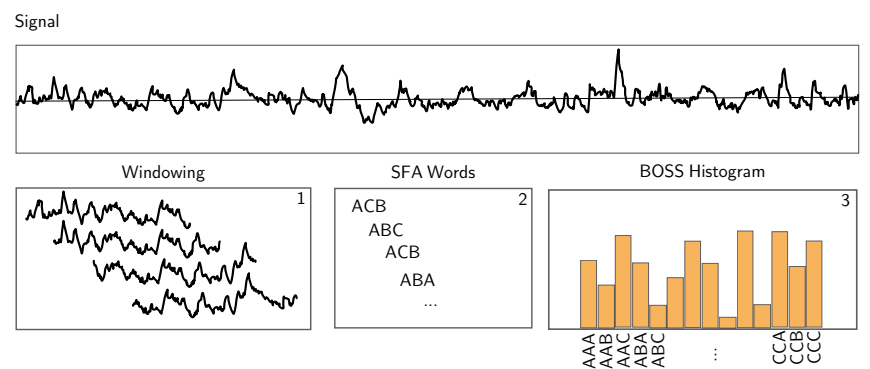

Figure 2: BOSS Model is used for indexing and representation and transforms the time series into BOSS histograms

bag of words representation to later create a $k$-dimensional matrix.

The BOSS model describes time series as an unordered set of substructures using Symbolic Fourier Approximation (SFA) words. The BOSS flow model is represented as follows (see Fig 2 [Schäfer, 2015]): parameters definition, windowing, SFA and histograms aggregation and reduction.

\section{Representation and indexing}

The goal of this step is to transform each time series to a new representation based on a vocabulary extracted from the behavior of the data in the frequency domain.

This transformation has a set of parameters that need to be tuned in order to find the optimal configuration: length of the windows, the number of Fourier coefficients for a window and the number of letters for the word representation.

For each time series, a set of fixed-size windows are generated using a sliding window of length $w$. The first window begins at 0 and ends in the position $w$, the second one will offset one position ending at position $w+1$ and so on until the end of the series is reached. This process generates $x-w+1$ windows. The result will be transformed into a vocabulary where each window represents a word. The length of the words are defined by the quantization of the Fourier coefficients of the window as explained in the following paragraphs.

The transformation of the windows into a vocabulary uses the SFA method, which aims to simplify information by removing the unnecessary data and keeping only the most representative characteristics. The SFA performs three steps to achieve its goal: approximation, quantization and words computation.

The SFA uses a discretization method based on the Momentary Fourier Transform (MFT), for the approximation process. The MFT targets to keep the critical data extracting the Fourier coefficients of the signal [Albrecht et al., 1997]. This method allows to incrementally compute the first $f$ Fourier coefficients of a sliding window in a series in efficient manner.

The idea behind this discretization is to decompose the time series into two basic type of functions [Schäfer and Högqvist, 2012]. The former consists in identifying slow changes in the data, while the latter identifies rapid changes. For the approximation, those with slow changes are enough for a fair description of the signal. These types of functions also provide a smoother signal with low pass filtering.
The decomposition represents a time series by its Fourier coefficient. The magnitude of the coefficient represents the amplitude of the signal. The proposed approximation uses only the first $f$ coefficients. The first Fourier coefficient can be optionally discarded because it stands for the mean value of the signal, obtaining this way offset invariance.

The quantization step reduces the granularity of the data by dividing the values of the Fourier coefficients into a histogram of equal frequency bins [Gurajada and Srivastava, 1991] and mapping each coefficient to its range. To define these histograms, a number of bins is defined as a parameters, representing the letters of the discretization alphabet that will be used for computing the words representing the windows extracted from the time series. Each position of the Fourier coefficients is discretized separately. This is done by computing multiple coefficient binning (MCB), and it aims to minimize the lost information when performing the discretization process.

The discretization works as a map that contains intervals of numeric values per each coefficient. The outcome of the SFA is a word of $f$ letters per window and a set of words per time series. In order to avoid a bias due to large periods of stable signal, the BOSS model reduces the length of the vocabulary by numerosity reduction, removing identical consecutive words.

The final stage of the BOSS model is to transform the vocabulary for a time series into a histogram of relative frequencies of words. This transforms the series into a vector, so we can compare different series using different similarity measures. The main advantage of this final transformation is to allow the comparison of time series of different lengths.

\section{Similarity Measure and Space Embedding}

The next step is to evaluate how similar are the sets of time series. From the possible similarity functions that can be defined, the one that better fits the model is the cosine similarity measure. The output of this process is an affinity matrix that will be further transformed before clustering the data.

For this model, a Spectral Embedding [Strange and Zwiggelaar, 2014] is applied to the affinity matrix to embed the data in a metric space and to enhance the relevant characteristics by using a non linear transformation. This embedding transforms the affinity matrix into a $k$-dimensional matrix. The number of dimensions $k$ is part of the configuration parameters. For this paper, the transformation was limited at most to three dimensions.

\section{Clustering}

A Bayesian Gaussian Mixture Model (BGMM) with Dirichlet priors [Sanjay-Gopal and Hebert, 1998; Li and Mihaylova, 2017] is used for partitioning the data represented by the $k$ dimensional data matrix. A Gaussian mixture model is a probabilistic model that assumes all the data points are generated from a mixture of a finite number of Gaussian distributions with unknown parameters. Each cluster is formed with a set of points that shape a Gaussian distribution using a Expectation Maximization (EM) algorithm. The use of a Dirichlet prior includes the determination of the number of clusters in the optimization process. 


\begin{tabular}{|l|l|l|l|l|}
\hline \multicolumn{5}{|c|}{ Set of variables and evaluation per scenario } \\
\hline Description & S.1.1 & S.1.2 & S.2.1 & S.2.2 \\
\hline Window size & 135 & 128 & 131 & 131 \\
Word length & 7 & 4 & 6 & 4 \\
No. of coeff. & 2 & 2 & 2 & 8 \\
Dimension & 3 & 3 & 2 & 3 \\
\hline Adj. Rand index & 0.90 & 0.93 & 0.93 & 0.93 \\
Silhouette index & 0.52 & 0.43 & 0.50 & 0.47 \\
No. clusters & 4 & 4 & 3 & 5 \\
\hline
\end{tabular}

Table 1: Results of the best two configurations of initial parameters per scenario and their evaluation.

The EM for mixture models consists of two steps. The first step calculates the expectation of the component for each data point given by the model parameters. The second phase maximizes the expectations calculated in the previous step concerning the model parameters. Then these two steps are repeated until the result converges.

\section{Evaluation}

To assess the quality of the clustering, it is necessary to apply some evaluation techniques. This approach considers two techniques: $(i)$ the adjusted rand index (ARI) [de Vargas and Bedrega, 2013] to measure the stability of the clustering to random initialization and (ii) the Silhouette index (SI) [Verma et al., 2015].

\subsection{Scenarios}

The work is based on the recognition of the patterns of the forces applied by the users to the $i$-Walker while walking in an indoor hallway. From the six forces recorded by the $i$-Walker, the vertical ones are the most related to the individuals' compensation strategies. Therefore, this study will examine two scenarios. The first model will be provided with the six forces to find if the transversal and longitudinal forces add relevant information. The second one will consider only the vertical forces to study whether the vertical and longitudinal forces add noise instead of contributing to the results.

\section{Experiments}

This section presents the results obtained by applying the model clustering approach on both scenarios. The goal of the model is to find the set of parameters that provide the best clustering. Thus, the results are presented in two parts: the performance comparison of the different sets of parameters and the knowledge discovery.

Table 1 lists the best two sets of parameters per scenario with the highest likelihood along with their validation indexes and their number of clusters, all of them worthy to analyze for pattern recognition.

The knowledge discovery was carried out by visualizing the behaviour of patterns through several plots. It was necessary to select statistical variables that better represented the problem. A finding is identified if the comparison of those variables enhances the visualization of the groups of clusters. The selected variables are (i) the cluster, (ii) age, (iii, $i v, v$ ) the mean, standard deviation and coefficient of variation of the considered forces, and (vi) the mean of the linear speed.
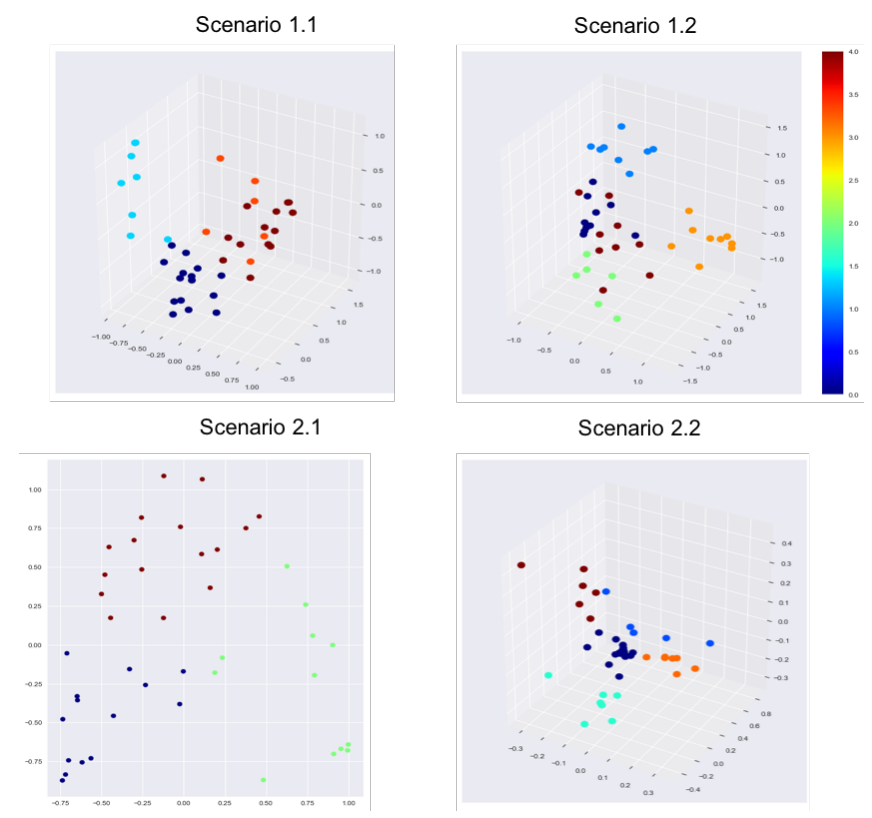

Scenario 2.2

Figure 3: Representation of the affinity matrix per scenario and the resulting BGMM Clustering

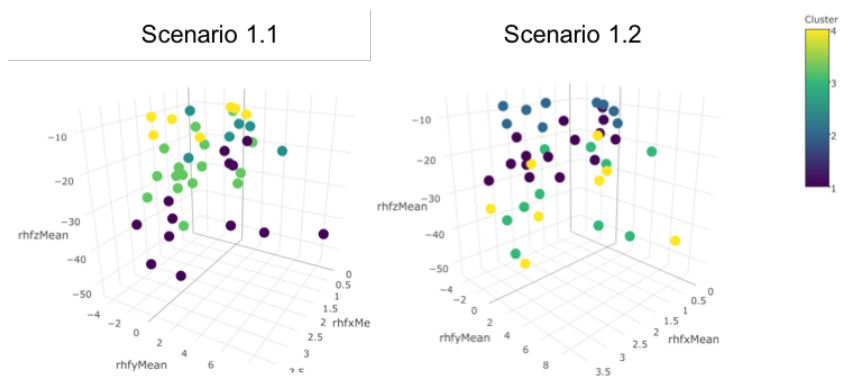

Figure 4: 3D plot of the vertical, longitudinal and transversal forces per cluster. The clustering is identified mainly over the axis of the vertical force.

Fig 3 shows the central clustering, the one made over the embedded affinity matrix and at which the BGMM identifies the distributions. Even though the two-dimensional spectral matrix presents an adequate grouping, the three-dimensional matrix can extract more features, and it is reflected in the number of clusters formed.

The next step is to identify if clustering visualization is preserved by comparing the selected variables. Fig 4 depicts the relationship between the $X, Y$ and $Z$ forces of the first scenario's set of parameters and it is noticeable that, indeed, the vertical forces have a notorious influence over the clustering.

Fig 5 shows that, if the standard deviation of the vertical force is added, the segments of clusters are more noticeable, which means that the variability measure is a factor that influences the clustering. Therefore, the proposed model meets its goal at considering the changes of the forces in the feature extraction. If the coefficient of variation is added, only the scenario 1.1 presents an improvement of the visualization as 

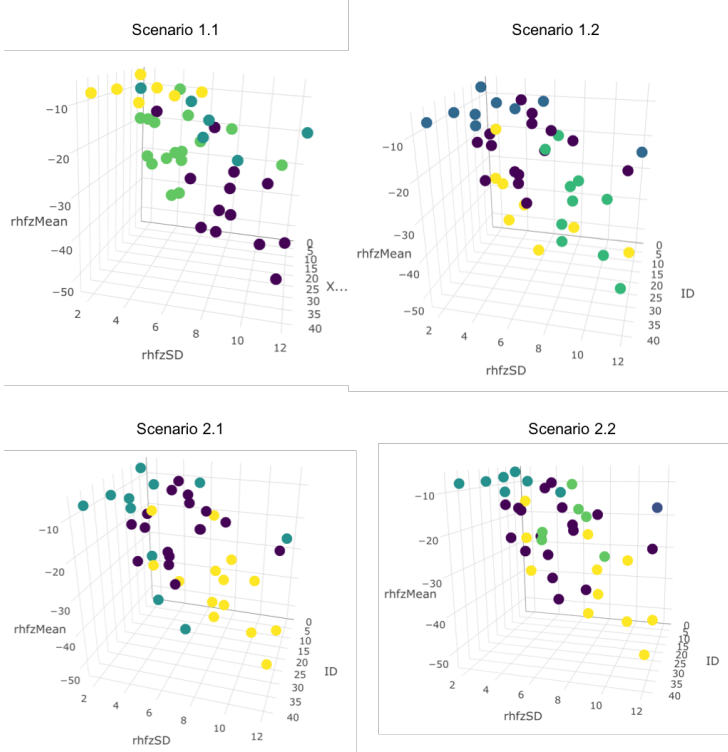

Figure 5: Comparison of the vertical force and its variability per cluster. It can be seen that the variability is directly related with the magnitude of $\mathrm{z}$-force.

observed in Fig 6.

When comparing the mean of the vertical force $v s$. the linear speed, it can be observed in Fig 7 that this variable is essential for the scenario 1.2. Moreover, if the age of the person is added as well, there is a critical pattern that splits the clusters by age and speed as shown in Fig 7.

\subsection{Clustering description}

This section presents a comparison of the clustering results among scenarios. The characteristics considered are $(i)$ the number of persons that contain each cluster per range of age, (ii) the average vertical force, (iii) the standard deviation of the vertical force variability and (iv) the average linear speed.

Table 2 describes the scenario 1.1. From the four clusters, clusters one and four have the most notorious distribution of people per groups, older adults and young people respectively. The average forces are by far the most critical difference between each cluster as well as its variability.

The scenario 1.2, described in Table 3, has a more radical distribution of people: clusters one and three mainly contain the younger individuals and cluster two and four the older ones. These clustering characteristics are remarkably interesting. The clusters that contain the younger participants have similar average speed but a notorious difference in their average vertical force. On the contrary, the groups including the older adults have similar average $Z$-force and variability, but they present a vast difference in the average speed. This contrast is enhanced because the primary descriptor of cluster four is indeed the velocity, it contains the eight individuals with the lowest average speed, while the primary descriptor of the group two is the average $Z$-force. An observation about the clusters of young people is that even though all of them have similar characteristics, due to the lack of experience of using a rollator they tend to change their walking behaviour.

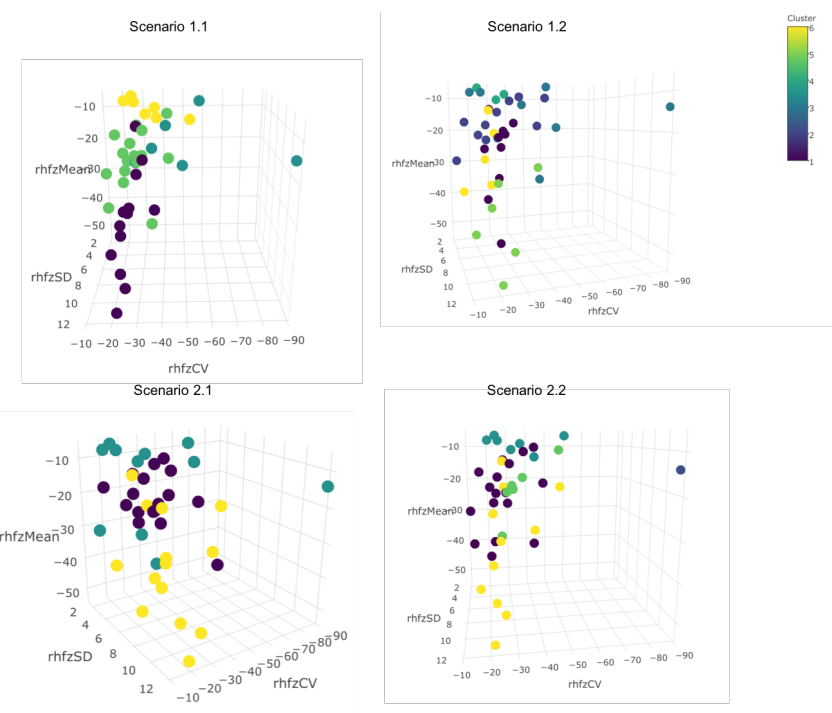

Figure 6: Comparison of the average, variability and coefficient of variation of the vertical force per cluster.

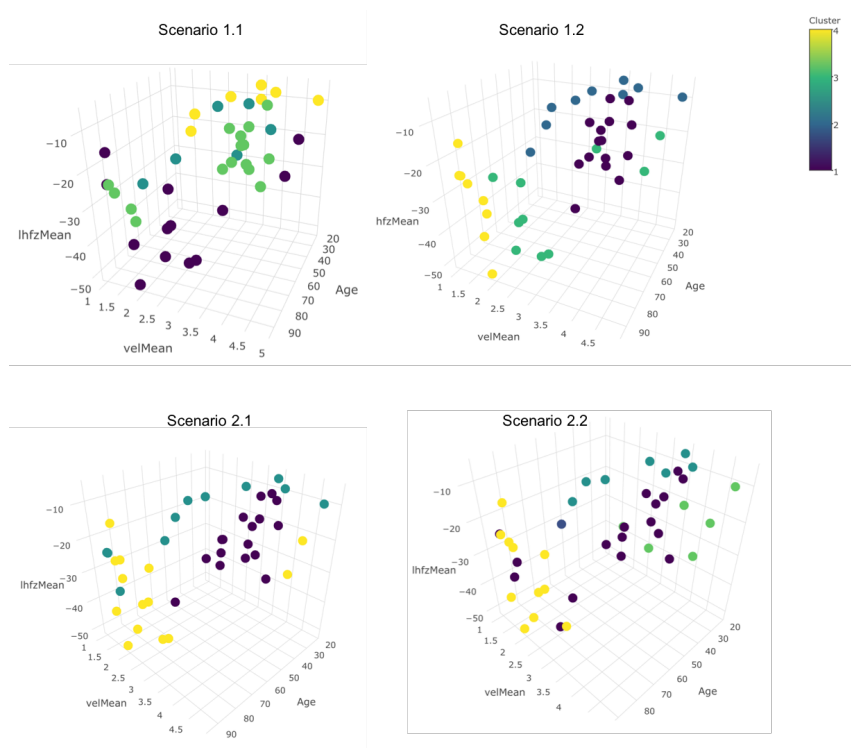

Figure 7: Comparison of the vertical force, its variability and the linear speed per cluster. The axis of the linear speed presents a tendency in the scenario 1.2 and 2.2 . 


\begin{tabular}{|l|l|l|l|l|}
\hline \multicolumn{5}{|c|}{ Clustering of Scenario 1.1 } \\
\hline Description & Clust.1 & Clust.2 & Clust.3 & Clust.4 \\
\hline Under 65 years & 2 & 3 & 7 & 5 \\
65-80 years & 0 & 2 & 5 & 2 \\
Above 80 years & 11 & 1 & 4 & 0 \\
Avg. z-force(N) & 34.7 & 15.3 & 21.5 & 9.1 \\
Z-force sd.(N) & 8.2 & 7.9 & 5.7 & 2.7 \\
Avg.speed(m/s) & 0.75 & 0.83 & 0.84 & 0.95 \\
\hline
\end{tabular}

Table 2: Scenario 1.1 clustering description. The vertical force and its variability are the two major differentiator factors

\begin{tabular}{|l|l|l|l|l|}
\hline \multicolumn{5}{|c|}{ Clustering of Scenario 1.2 } \\
\hline Description & Clust.1 & Clust.2 & Clust.3 & Clust.4 \\
\hline Under 65 years & 9 & 3 & 5 & 0 \\
65-80 years & 5 & 0 & 4 & 0 \\
Above 80 years & 1 & 7 & 0 & 8 \\
Avg. z-force(N) & 19.0 & 33.6 & 9.4 & 30.7 \\
Z-force sd.(N) & 5.6 & 8.8 & 4.1 & 7.0 \\
Avg.speed(m/s) & 0.99 & 0.85 & 0.92 & 0.42 \\
\hline
\end{tabular}

Table 3: Scenario 1.2 clustering description. All groups have a visible difference in the exerted forces, the variability and the speed.

Thus, the algorithm classifies them into two categories.

In Table 4, it can be observed that the scenario 2.1 has three clusters. In two of them, the distribution between young and elderly people is visible, but cluster two has a mix of both. The last scenario is presented in Table 5, which has been divided into five clusters, but the last one contains only one person. Therefore, it can be said that it has four relevant clusters and an outlier individual. From those four, again two clusters contain the young and healthy people, while the fourth cluster represents the elderly group. In fact, the distribution of clusters is quite similar to the one obtained in scenario 1.2 in terms of the applied vertical forces, however the average speed is different between scenarios, as well as the distribution of people aged $80+$ years old.

The configuration of the scenario 1.2 has the bests results because it presents a better categorization per groups of age and because both variables, the speed (supported by the literature) and the forces applied (proposed by the presented approach) were notorious differentiators between the clusters. This scenario also leads to thinking that the longitudinal and transversal forces do not add noise to the results, on the contrary, they enrich them.

\begin{tabular}{|l|l|l|l|}
\hline \multicolumn{4}{|c|}{ Clustering of Scenario 2.1 } \\
\hline Description & Clust.1 & Clust.2 & Clust.3 \\
\hline Under 65 years & 11 & 4 & 2 \\
65-80 years & 5 & 4 & 0 \\
Above 80 years & 1 & 3 & 12 \\
Avg. z-force(N) & 18.7 & 14.8 & 8.5 \\
Z-force sd.(N) & 5.6 & 8.8 & 4.1 \\
Avg.speed(m/s) & 0.98 & 0.77 & 0.70 \\
\hline
\end{tabular}

Table 4: Scenario 2.1 clustering description. The clusters have differences mainly in the average vertical force and its variability

\begin{tabular}{|l|l|l|l|l||l|}
\hline \multicolumn{7}{|c|}{ Clustering of Scenario 2.2 } \\
\hline Description & Cl.1 & Cl.2 & Cl.3 & Cl.4 & Cl.5 \\
\hline Under 65 yrs & 9 & 4 & 4 & 0 & 0 \\
65-80 yrs & 3 & 2 & 3 & 0 & 1 \\
Above 80 yrs & 5 & 0 & 0 & 11 & 0 \\
Av.z-force(N) & 22.5 & 20.2 & 8.8 & 33.7 & 13.8 \\
z-force sd(N). & 5.7 & 5.8 & 3.0 & 8.9 & 14.3 \\
Av.speed(m/s) & 1.18 & 0.88 & 0.58 & 0.68 & 0.08 \\
\hline
\end{tabular}

Table 5: Scenario 2.2 clustering description. The clusters have differences in the three variables presented.

\section{Conclusion and Future Work}

This paper proposes an analysis methodology that can classify an individual's walking-age based on the 3 minutes Walking Test using the $i$-Walker. A database of signal recordings was built to test the methodology applying data preprocessing, clustering, and classification as an automated process. The experimental results determine a suitable configuration of initial parameters that distinct walking-age groups according to the forces exerted by the individual on the $i$-Walker and their walking speed. Therefore, there is an evidence that the compensation strategies for walking can influence the person's gait and that it is also a useful indicator metric to measure health. Another conclusion is that the $i$-Walker is a practical, safe and efficient tool that can be easily implemented to collect high-quality data successfully. In addition, results regarding the relationship between age and walking speed are coherent with the literature [Montero-Odasso et al., 2005].

Although results are promising, the dataset used in this work fits for an exploratory analysis, however, statistically speaking, it falls into the realm of small datasets. As future work, it is expected to implement more walking tests to obtain a more extensive database to reinforce the analysis and findings. Also, it is recommended to include more elderly people to improve the quality of the results. The young group served as a baseline for this analysis, but according to [Ballesteros et al., 2016], rollators are useful for gait characterization as long as users need the device for ambulation.

It would be helpful to work with a specialist to provide additional information about the gait features that can be critical for the analysis and match them with other $i$-Walker descriptors. Aforementioned, it is also essential to develop an efficient analyzer of age-related problems, such as early detection of Alzheimer disease and other physical or mental issues.

\section{Acknowledgments}

The authors are partially supported by the project Rehabilitación personalizada y adaptativa en tratamientos post-ictus: el i-Walker: (TEC2014-56256-C2-2-P). Prof. Ulises Cortés is a member of Sistema Nacional de Investigadores (SNI-III), México. 


\section{References}

[Albrecht et al., 1997] S. Albrecht, I. Cumming, and J. Dudis. The momentary fourier transformation derived from recursive matrix transformations. 13th International Conference on Digital Signal Processing Proceedings, 7 1997.

[Ballesteros et al., 2016] J. Ballesteros, C. Urdiales, A.B Marinez, and J.H. van Dieen. On gait analysis estimation errors using force sensors on a smart rollator. Sensors, 16(11), 112016.

[Barban et al., 2017] F. Barban, M. Annicchiarico, R.and Melideo, A. Federici, M.G. Lombardi, S. Giuli, C. Ricci, F. Adriano, I. Griffini, M. Silvestri, M. Chiusso, S. Neglia, S. Ariño-Blasco, R. Cuevas Perez, Y. Dionyssiotis, G.s Koumanakos, M. Kovačeić, N. MonteroFernández, O. Pino, N. Boye, U. Cortés, C. Barrué, A. Cortés, P. Levene, S. Pantelopoulos, R. Rosso, J.A. Serra-Rexach, A. Maria Sabatini, and C. Caltagirone. Reducing fall risk with combined motor and cognitive training in elderly fallers. Brain Sciences, 7(2), 2017.

[CDC, 2016] Center of Disease Control and Prevention CDC. Falls are leading cause of injury and death in older americans, 2016. https://www.cdc.gov/media/releases/2016/p0922-olderadult-falls.html.

[Cortés et al., 2012] U. Cortés, A. Martínez-Velasco, C. Barrué, and R. Annicchiarico. AI based fall management services - the role of the i-Walker in I-DONTFALL. In Advances in Artificial Intelligence - $11^{\text {th }}$ Mexican Int. Conf. on Artificial Intelligence, MICAI 2012, San Luis Potosí, Mexico., pages 395-406, 2012.

[Cortés et al., 2016] A. Cortés, J. Béjar, C. Barrué, A.B. Martínez, and U. Cortés. Assessing falling risk in elderly with the ten meter walking test: A machine learning approach. In Artificial Intelligence Research and Development - Proc. of the $19^{\text {th }}$ Int. Conf. of the Catalan Association for Artificial Intelligence, CCIA 2016, Barcelona, Spain., pages 227-232, 2016.

[de Vargas and Bedrega, 2013] R. de Vargas and B. Bedrega. A way to obtain the quality of a partition by adjusted rand index. Workshop-School on Theoretical Computer Science (WEIT), 102013.

[Dolatabadi et al., 2017] E. Dolatabadi, A. Mansfield, K. Patterson, B. Taati, and A. Mihailidis. Mixture-model clustering of pathological gait patterns. IEEE Journal of Biomedical and Health Informatics, 21(5), 92017.

[Giuliani et al., 2012] B. Giuliani, U. Cortés, A.B. MartínezVelasco, C. Barrué, and R. Annicchiarico. The role of iWalker in post-stroke training. In David Riaño, Eva Onaindia, and Miguel Cazorla, editors, Artificial Intelligence Research and Development - Proc. of the $15^{\text {th }}$ Int. Conf. of the Catalan Association for Artificial Intelligence, CCIA 2012, volume 248 of Frontiers in Artificial Intelligence and Applications, pages 133-142. IOS Press, 2012.
[Gurajada and Srivastava, 1991] A.P. Gurajada and J. Srivastava. Equidepth partitioning of a data set based on finding its median. Symposium on Applied Computing, 21991.

[IDF, 2010] Integrated prevention and Detection sOlutioNs Tailored to the population and Risk Factors associated with FALLs. http://www.idontfall.eu/, 2010.

[Jin et al., 2014] B. Jin, T. Hoai Thu, E. Baek, S. Sakong, J. Xiao, T. Mondal, and M. Deen. Walking-age analyzer for healthcare application. IEEE Journal of Biomedical and Health Informatics, 18(3), 52014.

[Li and Mihaylova, 2017] Z. Li and L.S. Mihaylova. Autonomous flame detection in videos with a dirichlet process gaussian mixture color model. IEEE Transactions on Industrial Informatics, PP(99), 112017.

[Montero-Odasso et al., 2005] M. Montero-Odasso, M. Schapira, E.R. Soriano, M. Varela, R. Kaplan, L. A. Camera, and L. M. Mayorga. Gait velocity as a single predictor of adverse events in healthy seniors aged 75 years and older. The Journals of Gerontology Series A: Biological Sciences and Medical Sciences, 60(10):1304-1309, 2005.

[Moreno et al., 2015] J. Moreno, A. Cortés, C. Barrué, and U. Cortés. A methodology for pattern detection in gait behaviour using the i-walker. In Artificial Intelligence Research and Development - Proc. of the $18^{\text {th }}$ Int. Conf. of the Catalan Association for Artificial Intelligence, CCIA 2015, volume 277 of Frontiers in Artificial Intelligence and Applications, pages 287 - 290. IOS Press, 2015.

[Nations, 2017] United Nations. World population ageing 2017 - highlights. World Population Ageing, page 5, 2017.

[Sanjay-Gopal and Hebert, 1998] S. Sanjay-Gopal and T.J. Hebert. Bayesian pixel classification using spatially variant finite mixtures and the generalized em algorithm. IEEE Transactions on Image Processing, 7(7), 71998.

[Schäfer and Högqvist, 2012] P. Schäfer and M. Högqvist. SFA: A symbolic fourier approximation and index for similarity search in high dimensional datasets. Proceedings of the 15th International Conference on Extending Database Technology, 32012.

[Schäfer, 2015] P. Schäfer. The BOSS is concerned with time series classification in the presence of noise. Data Mining and Knowledge Discovery, 29(6), 112015.

[Strange and Zwiggelaar, 2014] H. Strange and R. Zwiggelaar. Open Problems in Spectral Dimensionality Reduction. Springer, Alberystwyth, 2014.

[Verma et al., 2015] N. Verma, E. Dutta, and Y. Cui. Hausdorff distance and global silhouette index as novel measures for estimating quality of biclusters. Int. Conf. on Bioinformatics and Biomedicine, BIBM, 2015. 